\title{
Genetic characterization of the silkworm Bombyx mori by simple sequence repeat (SSR)-anchored PCR
}

\author{
K. DAMODAR REDDY $\dagger$, J. NAGARAJU*†+ \& E. G. ABRAHAM $\dagger$ \\ $\dagger$ Seribiotech Research Laboratory, Central Silk Board, No. 8, West of Chord Road, Bangalore-560 086, India and \\ $\$$ Centre for DNA Fingerprinting and Diagnostics, CCMB Campus, Uppal Road, Hyderabad-500 007, India
}

\begin{abstract}
Thirteen diverse strains of the silkworm Bombyx mori were analysed using the simple sequence repeat anchored polymerase chain reaction (SSR-anchored PCR) or Inter-SSR-PCR (ISSR-PCR). A set of four $5^{\prime}$-anchored and two $3^{\prime}$-anchored repeat primers amplified a total of 239 bands out of which 184 $(77 \%)$ were polymorphic. The $5^{\prime}$-anchored primers revealed more distinct polymorphic markers than the $3^{\prime}$-anchored primers and the ISSR-PCR method showed greater variability than RAPDs. The strain-specific pattern was shown to be inherited and segregated in a Mendelian fashion. A dendrogram constructed using the UPGMA method revealed two distinct groups, one comprising nondiapausing and one comprising diapausing strains. These results suggest that the ISSR-PCR method is potentially useful for genetic fingerprinting of silkworm genotypes and as a mapping tool in the silkworm.
\end{abstract}

Keywords: Bombyx mori, genome fingerprinting, ISSR-PCR.

\section{Introduction}

Eukaryotic genomes are densely interspersed with tandem repeats termed microsatellites or simple sequence repeats (SSR) (Hamada et al., 1982; Tautz \& Renz, 1984; Tautz et al., 1986; Weber \& May, 1989). These are short DNA sequence motifs (usually $2-5 \mathrm{bp}$ long) that occur at multiple sites (Beckmann \& Weber, 1992; Wang et al., 1994; Field \& Wills, 1996) and reveal a high degree of allelic diversity which can be typed via the polymerase chain reaction (Schlotterer et al., 1991). Analysis of SSRs provides a codominant, highly reproducible and genetically informative marker system. However, this method is quite labour-intensive because complete sequence information is necessary to design primers for the single-copy sequences flanking the microsatellites being scored.

As PCR technology finds increased use in genetic analysis, additional novel variations of this technique are emerging. PCR analysis using anchored simple sequence repeat primers has gained attention recently as an alternative means of characterizing complex genomes. This approach employs oligonucleotides based

*Correspondence. J. Nagaraju, Centre for DNA Fingerprinting and Diagnostics, CCMB Campus, Uppal Road, Hyderabad - 500 007, India. E-mail: jnagaraju@mailcity.com on an SSR anchored at either the $5^{\prime}$ or $3^{\prime}$ end with two to four purine or pyrimidine residues, to initiate PCR amplification of genomic segments flanked by inversely orientated, closely spaced microsatellite repeats (Zietkiewicz et al., 1994). The PCR products thus generated reveal multiple polymorphic products which can be resolved on a single high resolution polyacrylamide gel. The Inter-SSR-PCR (ISSR-PCR) strategy is especially attractive because it avoids the need to carry out costly cloning and sequencing inherent in the original microsatellite-based approach. Moreover, locus-specific probes and microsatellite-containing sequences of interest can be developed by isolating and cloning or reamplifying individual bands (Wu et al., 1994; Zietkiewicz et al., 1994). ISSR-PCR has been profitably used for genetic linkage analysis of various plant species (Kantety et al., 1995; Charters et al., 1996; Provan et al., 1996; Tsumura et al., 1996). Furthermore, the technique of ISSR amplification is sensitive enough even to differentiate between closely related individuals (Zietkiewicz et al., 1994).

The silkworm Bombyx mori has emerged as a lepidopteran molecular model system for diverse biological studies, including genetics, development and physiology (Goldsmith, 1995), in addition to retaining its economic importance in silk production. The silkworm has a large number of geographical races and 
inbred lines which show substantial variation for a large number of quantitative traits. The traditional breeding activities, involving hybridizations between members of elite groups, are adding new varieties every year. At present, in the silkworm, traits such as cocoon shape, cocoon colour, silk fibre length, larval duration, together with many other ethological traits, are used to differentiate varieties. The selection of parental strains for a breeding programme is based on these characteristics. But the silkworm varieties, particularly those which have been bred from crosses involving many varieties, cannot be distinguished unambiguously by the use of conventional characteristics. It is thus apparent that the use of molecular markers could provide a solution to the problem, by providing unique DNA profiles. Such varietal DNA profiles would be useful in producing reliable estimates of genetic diversity, for the selection of parents for the development of elite hybrids, and to protect silkworm breeders' rights. Varietal-specific DNA markers could also provide additional markers for the ongoing silkworm genome mapping programme. If economically important traits are found to have close linkage with the DNA markers, the latter could also be used in marker-assisted selection. Attempts have already been made to fingerprint the diverse silkworm genotypes and establish their genetic relationships using defective transposons (Tamura et al., 1993), RAPDs (Nagaraja \& Nagaraju, 1995), a Bkm-derived probe (Nagaraju et al., 1995) and SSRs (Reddy et al., 1999). In the present study, we report the feasibility of using the ISSR-PCR method to analyse the diverse genotypes of silkworm in order to augment marker resources for silkworm genetic analysis.

\section{Materials and methods}

\section{Silkworm strains}

Six diapausing $\left(\mathrm{Hu}_{204}, \mathrm{KA}, \mathrm{NB}_{1}, \mathrm{NB}_{7}, \mathrm{NB}_{18}, \mathrm{NB}_{4} \mathrm{D}_{2}\right)$ and seven nondiapausing (C. nichi, Daizo, Gungnong, Moria, Nistari, Pure Mysore and Sarupat) silkworm strains were used in the present study. $F_{1}$ offspring were obtained by crossing a male of $\mathrm{NB}_{1}$ with a Nistari female. $F_{1}$ hybrid offspring were mated among themselves to obtain $F_{2}$ offspring. The details of the characteristics of the silkworm strains used in the present study have been reported earlier by Nagaraja \& Nagaraju (1995).

\section{DNA extraction}

Genomic DNA from silk moths [13 strains, parents (Nistari and $\mathrm{NB}_{1}$ ), their $\mathrm{F}_{1}$ and $\mathrm{F}_{2}$ offspring], frozen in liquid nitrogen, was isolated by the method of Suzuki et al. (1972) as modified by Nagaraja \& Nagaraju
(1995). For genetic analysis of the silkworm strains, DNA from individual moths was isolated from $F_{1}, F_{2}$ offspring and their parents. In the case of the 13 silkworm strains, DNA was pooled from 10 moths of each of the representative strains.

\section{The anchored primers}

Seven oligonucleotides were utilized for this study: 3'-anchored repeat primers: (i) $(\mathrm{GT})_{8}(\mathrm{~A} / \mathrm{G}) \mathrm{R}$ (ii) $(\mathrm{GT})_{8}(\mathrm{~A} / \mathrm{G}) \mathrm{Y}$ and (iii) $(\mathrm{GT})_{8}(\mathrm{~A} / \mathrm{G}) \mathrm{TCY} ; 5^{\prime}$-anchored repeat primers: (i) GCT AGT GCT $(\mathrm{CA})_{7} \mathrm{Y}$ (ii) GCA CAT GCA R (TG) $)_{7}$ (iii) GAT GCT GAT R $(\mathrm{CA})_{7}$ and (iv) CAT GCA CAT (TG) ${ }_{7} \mathrm{Y}$, where R and Y represent purines and pyrimidines, respectively. These oligonucleotide primers were synthesized on a DNA synthesizer (Applied Biosystems), purified by PAGE and then desalted through an oligonucleotides purification cartridge (OPC). Oligonucleotides were 5 -end labelled using gamma ${ }^{32} \mathrm{P}-\mathrm{dATP}(5000 \mathrm{Ci} / \mathrm{mmol}$, BRIT, Hyderabad, India) and T4 polynucleotide kinase (Genei, India).

\section{PCR analysis}

Reaction mixtures $(20 \mu \mathrm{L})$ contained $10 \times$ PCR buffer $(500 \mathrm{~mm} \mathrm{KCl}, 100 \mathrm{~mm}$ Tris- $\mathrm{HCl}$ and $1.0 \%$ Triton X-100), $2 \mathrm{~mm}$ of each dATP, dGTP and dTTP and $0.75 \mathrm{~mm}$ $\mathrm{dCTP}, 10 \mu \mathrm{M}$ of oligonucleotide primer (a mixture of 1:4 of the labelled and cold primer), $3 \mathrm{~mm} \mathrm{MgCl}_{2}, 1$ unit of Taq polymerase (Amersham), and $20 \mathrm{ng}$ of template DNA. Samples were overlaid with $10 \mu \mathrm{L}$ of mineral oil and reactions were carried out on a Perkin Elmer 480 thermal cycler using the following reaction conditions. (i) $94^{\circ} \mathrm{C}$ for $2 \mathrm{~min}, 1$ cycle; (ii) $94^{\circ} \mathrm{C}$ for $30 \mathrm{~s}, 52^{\circ} \mathrm{C}$ for $45 \mathrm{~s}$, $72^{\circ} \mathrm{C}$ for $2 \mathrm{~min}, 27$ cycles; (iii) $72^{\circ} \mathrm{C}$ for $7 \mathrm{~min}, 1$ cycle. On completion of PCR, the reaction was stopped using $6 \mu \mathrm{L}$ of stop solution (95\% formamide, $20 \mathrm{~mm}$ EDTA, $0.05 \%$ bromophenol blue and $0.05 \%$ xylene cyanol).

Table 1 ISSR primers, and the number of polymorphic fragments amplified using each primer with 13 silkworm strains

\begin{tabular}{lcc}
\hline Anchored primer & $\begin{array}{c}\text { No. of } \\
\text { bands }\end{array}$ & $\begin{array}{c}\text { No. of } \\
\text { polymorphic bands }\end{array}$ \\
\hline$(\mathrm{GT})_{8}(\mathrm{~A} / \mathrm{G}) \mathrm{R}$ & 22 & 22 \\
$(\mathrm{GT})_{8}$ (A/G) Y & - & - \\
$(\mathrm{GT})_{8}(\mathrm{~A} / \mathrm{G})$ TCY & 57 & 35 \\
GCT AGT GCT $(\mathrm{CA})_{7} \mathrm{Y}$ & 42 & 33 \\
GCA CAT GCA R $(\mathrm{TG})_{7}$ & 55 & 50 \\
GAT GCT GAT R $(\mathrm{CA})_{7}$ & 45 & 29 \\
CAT GCA CAT $(\mathrm{TG})_{7} \mathrm{Y}$ & 18 & 15 \\
\hline
\end{tabular}




\section{Electrophoresis}

Four- $\mu \mathrm{L}$ aliquots were denatured at $75^{\circ} \mathrm{C}$ for $2 \mathrm{~min}$, chilled on ice and then loaded on a standard sequencing gel $(6 \%$ acrylamide, $8 \mathrm{~m}$ urea, $1 \times \mathrm{TBE}(89 \mathrm{~mm}$ Trisborate, 2 mм EDTA, $\mathrm{pH} 8.3$ ) and run at $900 \mathrm{~V}$ under constant power for $20 \mathrm{~h}$. After electrophoresis, gels were fixed for $2 \times 20 \mathrm{~min}$ in $10 \%$ glacial acetic acid, dried at room temperature and exposed to Kodak X-ray film for 2-10 $\mathrm{h}$ at room temperature.

\section{Data analysis}

Specific PCR products that were reproducible in successive amplifications were selected. Amplification

(a)

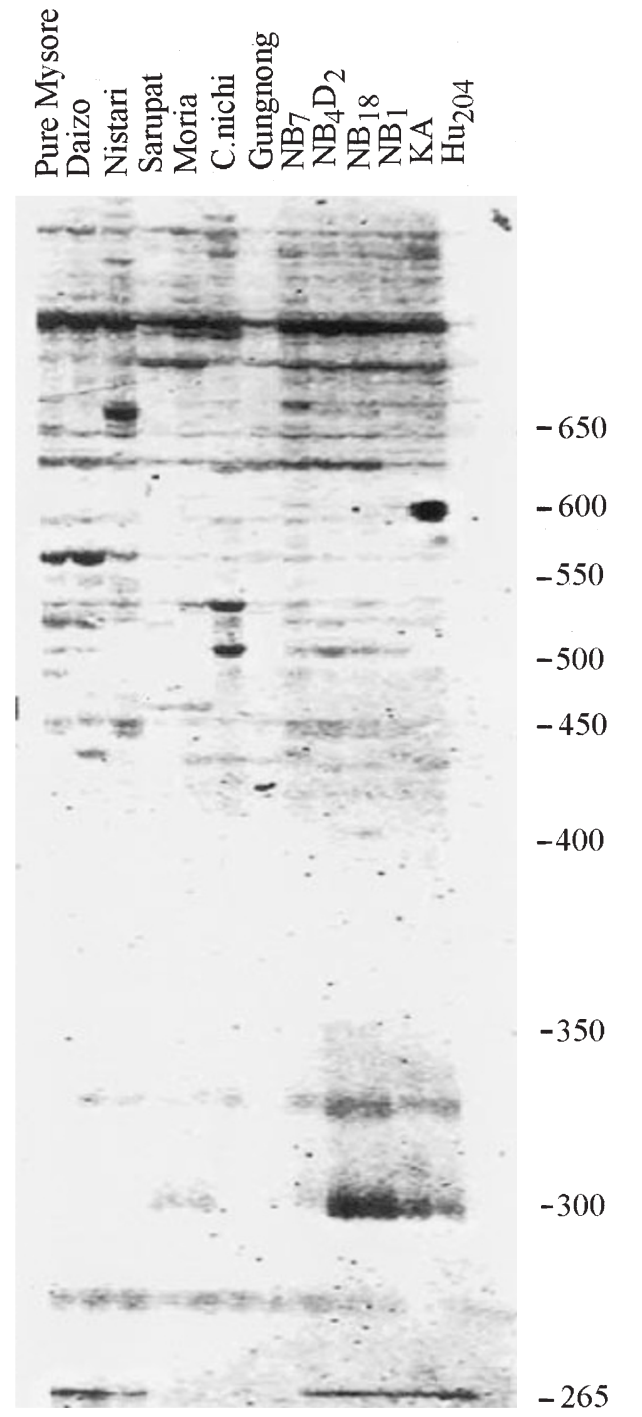

products were scored as 1 (present) or 0 (absent) for the strains, and similarity index matrices were generated based on the number of shared fragments. Similarity index $S=2 N_{\mathrm{ab}} /\left(N_{\mathrm{a}}+N_{\mathrm{b}}\right)$, where $N_{\mathrm{a}}$ and $N_{\mathrm{b}}$ represent the total number of bands present in lanes a and $b$, respectively, and $N_{\mathrm{ab}}$ is the number of bands shared by both lanes (Nei \& Li, 1979). The distance values were analysed using the unweighted pair group method analysis (UPGMA) program in WinBoot software (Yap $\&$ Nelson, 1996). The relationship between the 13 strains was portrayed graphically in the form of a dendrogram.

\section{Results}

To investigate the utility of ISSR-PCR in the genetic analysis of diverse silkworm strains, we tested three

(b)
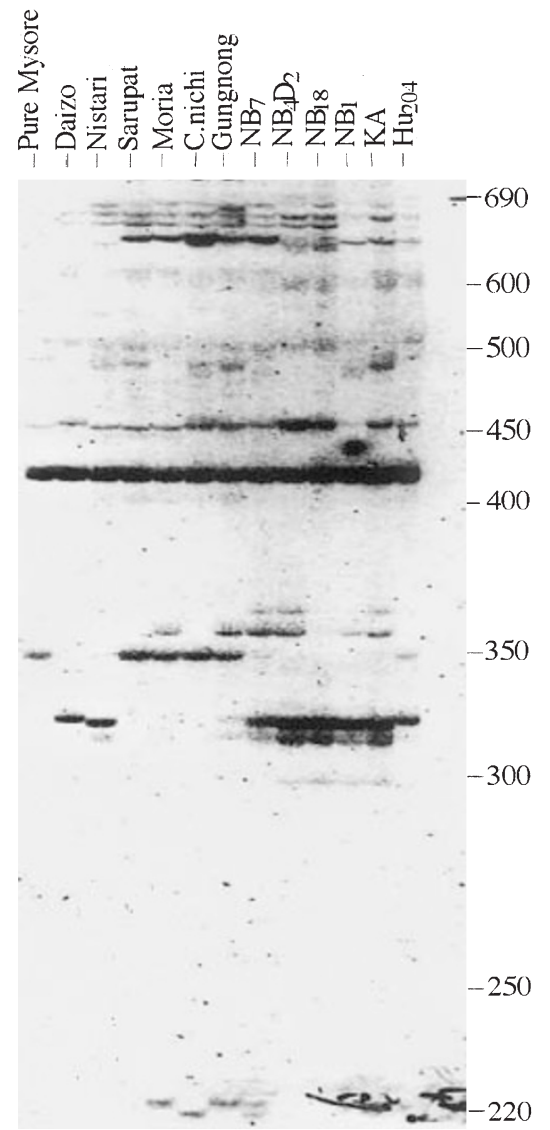
3 '-anchored primers and four 5'-anchored primers (Table 1). Of the seven primers tested, all but one 3 '-anchored primer $(\mathrm{GT})_{8}(\mathrm{~A} / \mathrm{G}) \mathrm{Y}$, amplified scorable PCR products. Inter-SSR amplification of the 13 silkworm strains with the six primers yielded a total of 239 bands, of which 184 (77\%) were polymorphic. The minimum and maximum numbers of bands observed were 18 (primer CAT GCA CAT $(\mathrm{TG})_{7} \mathrm{Y}$ ) and 57 (primer $\left.(\mathrm{GT})_{8}(\mathrm{~A} / \mathrm{G}) \mathrm{TCY}\right)$, respectively, with an average of 39.8. Figure 1(a) and 1(b) shows the amplification profiles generated across the 13 strains using the 3 '-anchored primer $(\mathrm{GT})_{8} \quad(\mathrm{~A} / \mathrm{G}) \quad \mathrm{TCY}$ and the 5 '-anchored primer GCA CAT GCA R (TG) ${ }_{7}$, respectively. Multiple bands varying in size from 200 to more than 1000 bp are seen in almost all strains. Such a large range of variability is perhaps caused by small insertions/deletions in the genomic region amplified between the microsatellite anchored regions. The abundant presence of the small retroposons, $B m_{1}$ (250 bp $450 \mathrm{bp})$ and $\mathrm{Bm}_{2}(125 \mathrm{bp})$, which account for 5-6\% of the haploid genome and are found inserted in most of the Bombyx genes cloned so far (Eickbush, 1995), could also perhaps explain such size variations. Further cloning and analysis of the amplified products in different strains may throw light on the distribution of these kinds of elements in silkworm strains of diverse origin. The two $3^{\prime}$-anchored primers $\left[(\mathrm{GT})_{8}(\mathrm{~A} / \mathrm{G}) \mathrm{R}\right.$ and $\left.(\mathrm{GT})_{8}(\mathrm{~A} / \mathrm{G}) \mathrm{TCY}\right]$ resulted in amplification of 79 products with an average of 39.5 bands, of which $72 \%$ were polymorphic. On the other hand the four $5^{\prime}$-anchored primers [GCT AGT GCT $(\mathrm{CA})_{7} \mathrm{Y}$, GAT GCT GAT R $(\mathrm{CA})_{7}$, GCA CAT GCA R (TG) ${ }_{7}$, and CAT GCA CAT $\left.(\mathrm{TG})_{7} \mathrm{Y}\right]$ generated 160 PCR products with an average of 40 bands, of which $79 \%$ were polymorphic. Although the two sets of anchored primers did not differ much in their informativeness, the $5^{\prime}$-anchored (a)

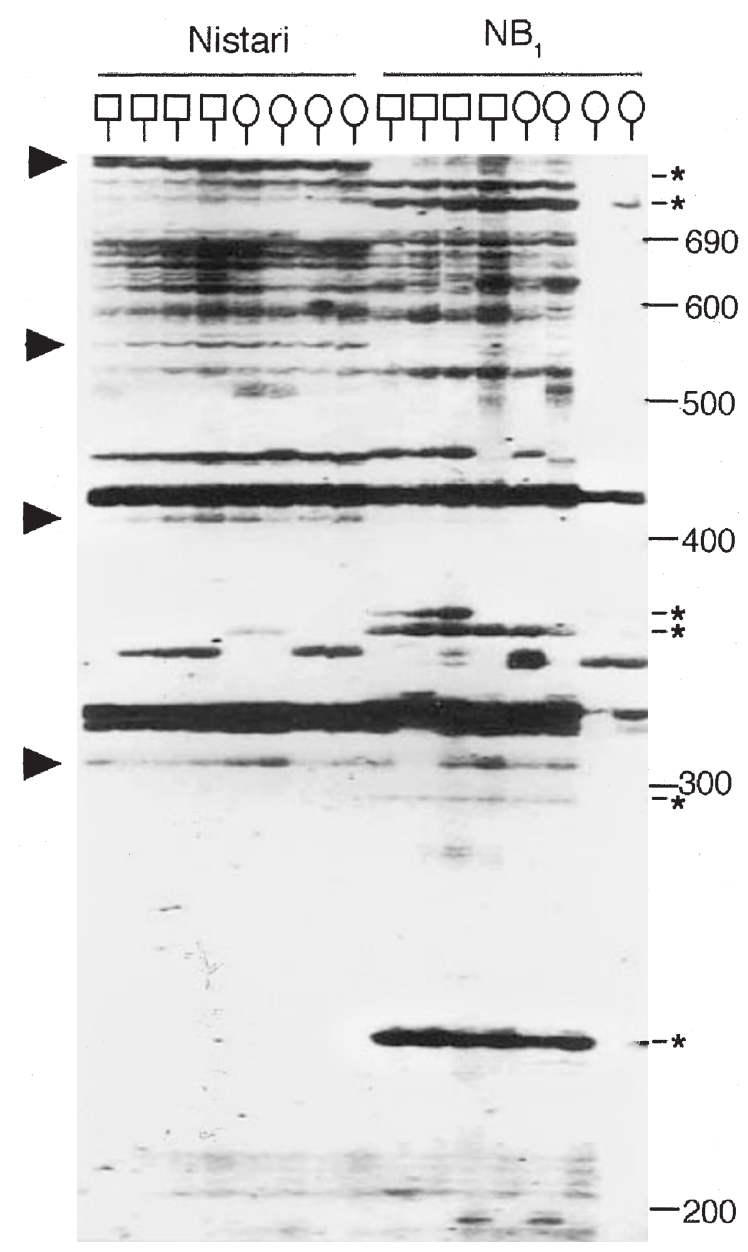

(b)

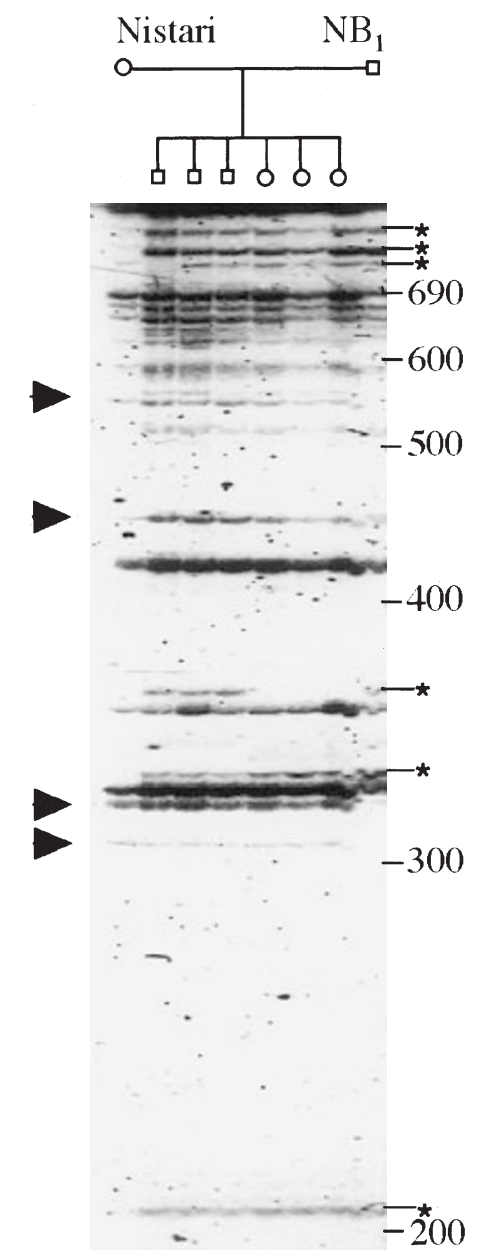

Fig. 2 Strain-specific pattern and codominant inheritance of ISSR markers generated by $5^{\prime}$-anchored primer GCT AGT GCT (CA) 7 Y. Amplification profiles from: (a) individuals of the Nistari and $\mathrm{NB}_{1}$ strains of silkworm; (b) $\mathrm{F}_{1}$ offspring and their parents. Arrows indicate strain-specific amplification products. The squares and circles represent males and females, respectively. 
primers resulted in more highly resolved distinct bands as compared to those of the $3^{\prime}$-anchored primers (see Fig. 1a,b). These results are consistent with the previous

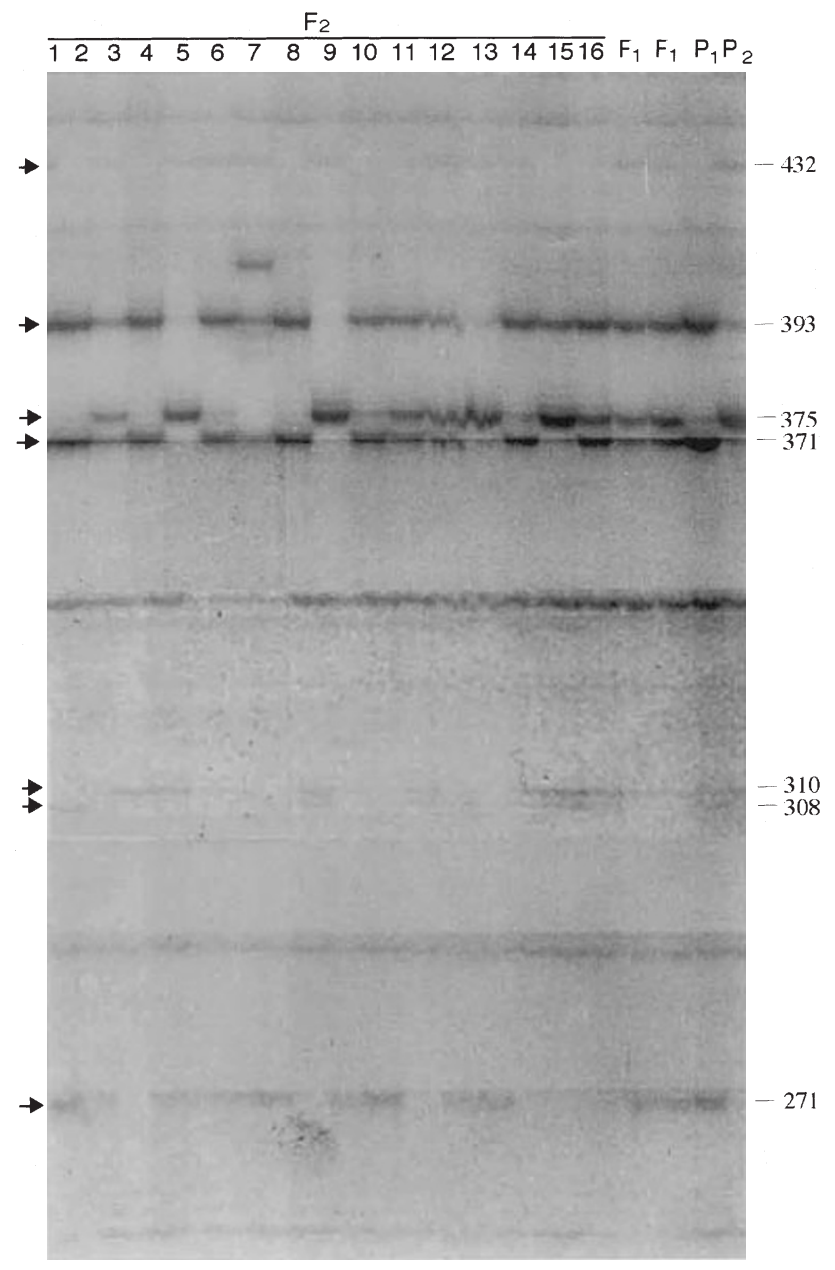

Fig. 3 Inheritance of ISSR loci in silkworm. ISSR profiles obtained using a primer CAT GCA CAT $(\mathrm{TG})_{7} \mathrm{Y}$ on two parental strains, $\mathrm{NB}_{1}\left(\mathrm{P}_{1}\right)$ and $\operatorname{Nistari}\left(\mathrm{P}_{2}\right)$, and their $\mathrm{F}_{1}$ and $\mathrm{F}_{2}$ offspring. The segregation of polymorphic loci is shown by arrows. studies (Zietkiewicz et al., 1994; Charters et al., 1996) which reported higher resolution and greater banding specificity of $5^{\prime}$-anchored primers. The complexity of patterns as well as the degree of polymorphism detected per single ISSR-PCR experiment were higher than those produced by RAPDs or by a Bkm probe (containing 66 copies of GATA repeats interspersed with TA repeats) (Nagaraja \& Nagaraju, 1995; Nagaraju et al., 1995; Promboon et al., 1995) in silkworm strains.

The amplification products originating from individuals of the same strain clearly displayed similar patterns, and strain-specific products could be unambiguously scored (Fig. 2a). For example, the $\mathrm{NB}_{1}$ strain had diagnostic amplification products of $233 \mathrm{bp}, 298 \mathrm{bp}$, $310 \mathrm{bp}, 335 \mathrm{bp}, 750 \mathrm{bp}$ and $780 \mathrm{bp}$. As could be seen, most of the markers were polymorphic within the strain. The Nistari strain revealed specific products of $417 \mathrm{bp}$, $549 \mathrm{bp}$ and $800 \mathrm{bp}$. Most of the markers were present in almost all the individuals. As we have examined only eight individuals in each of the strains, it is quite possible that we have not scored all the markers that are specific to a particular strain. The codominant inheritance of polymorphic bands was studied in a cross involving two diverse silkworm strains, Nistari and $\mathrm{NB}_{1}$, using the primer GCT AGT GCT (CA) 7 Y. Amplification shown in Fig. 2(b) revealed six polymorphic bands specific to $\mathrm{NB}_{1}$ and four to Nistari. The inheritance of these polymorphic bands was consistent with their behaviour as dominant markers, although some bands may actually represent allelic variants of the same locus.

Although the ISSR markers were reliable when analysed for their inheritance in $F_{1}$ offspring, we were interested to know how they segregated in the $F_{2}$ offspring. As the silkworm strains were not completely inbred (as shown in Fig. 2a) we analysed segregation of distinctly scorable markers in both $\mathrm{F}_{1}$ and $\mathrm{F}_{2}$ offspring of the Nistari and $\mathrm{NB}_{1}$ strains. In most of the cases, marker loci segregated in their expected ratio in the $\mathrm{F}_{2}$ offspring (3:1 or $1: 1)$, depending upon whether they were homo- or heterozygous in the parental strains (Fig. 3, Table 2).

Table 2 Mendelian segregation in silkworms of seven ISSR loci amplified by CAT GCA CAT (TG) ${ }_{7}$ Y

\begin{tabular}{|c|c|c|c|c|c|c|c|}
\hline Locus & Size (bp) & Zygosity & $\begin{array}{c}\text { No. of individuals } \\
\text { scored }\end{array}$ & Expected ratio & Observed ratio & $\chi^{2}$ & $P>$ \\
\hline 1 & 432 & Hetero & 50 & $25: 25$ & $25: 25$ & 0.00 & 0.99 \\
\hline 3 & 375 & Homo & 50 & $37.5: 12.5$ & $39: 11$ & 0.24 & 0.50 \\
\hline 4 & 371 & Homo & 50 & $37.5: 12.5$ & $39: 11$ & 0.24 & 0.50 \\
\hline 5 & 310 & Homo & 50 & $37.5: 12.5$ & $38: 12$ & 0.03 & 0.75 \\
\hline 7 & 271 & Homo & 50 & $37.5: 12.5$ & $35: 15$ & 0.67 & 0.10 \\
\hline
\end{tabular}

(C) The Genetical Society of Great Britain, Heredity, 83, 681-687. 


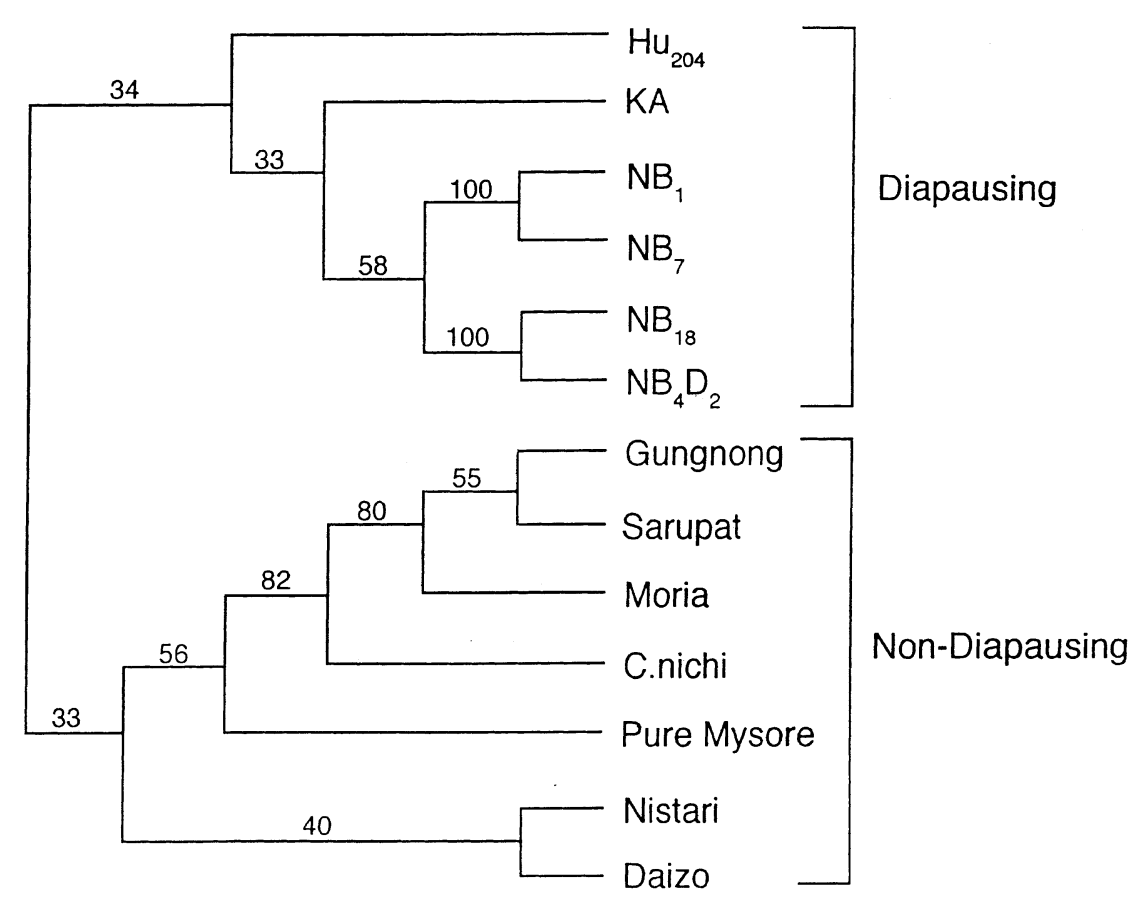

Fig. 4 UPGMA-derived dendrogram illustrating the relationships among 13 silkworm strains as inferred by ISSR-PCR analysis. Numbers on the nodes indicate the number of times a particular branch was recorded per 100 bootstrap replications following 1000 replications.

\section{Discussion}

The ISSR technique is fast, reliable, provides an almost inexhaustible supply of genetic markers, and requires very little template DNA. The Mendelian segregation of ISSR markers clearly underlines their immense utility in silkworm genetics. Hence it is an excellent way to map the genomes.

The ability to screen so many polymorphisms in a single assay makes the ISSR-PCR a technique of choice for a large-scale screening of germplasm. The pattern of similarity seen within a strain clearly underscores the utility of this method for inferring the genetic relationship of silkworm strains. A dendrogram generated by UPGMA analysis based on band sharing analysis resolved the 13 silkworm strains into two clusters, one comprising the six diapausing strains and the other with all of the seven nondiapausing strains (Fig. 4). The diapausing and nondiapausing characters of silkworm strains have a close relationship with their geographical distribution. The silkworm strains distributed in tropical countries like India, Vietnam, Thailand, China, etc., are nondiapausing in nature; in contrast, those distributed in temperate countries like Japan, France, Russia, Korea, etc., are diapausing. The nondiapausing strains are rapid breeders, attain lower body weight, secrete short and thicker silk fibre, and are resistant to high temperatures and viral diseases, whereas the diapausing strains have longer larval life, register higher body weight and secrete longer and thinner silk fibre, and are susceptible to high temperatures and viral pathogens. Hence, the distinct clustering of diapause and nondiapause silkworm strains reflects the geographical origin and, morphological, qualitative and quantitative traits associated with these two sets of strains. The dendrogram also reflected the pedigree history of the strains used. For example, $\mathrm{NB}_{18}$ and $\mathrm{NB}_{4} \mathrm{D}_{2}$ used in the study are derived from a common Japanese double hybrid and are grouped together. Similarly, Moria and Sarupat which show similar characteristics and are distributed in the state of Assam, are also grouped together. These observations are in agreement with the earlier results obtained with RAPDs (Nagaraja \& Nagaraju, 1995), the Bkm probe (Nagaraju et al., 1995) and SSRs (Reddy et al., 1999).

The fact that the ISSR method resolves diapause and nondiapause strain-specific amplification products makes it useful to augment the marker resources for the silkworm genome mapping programme. In the mapping strategy, the genetically diverse strains of silkworm, which possess highly contrasting qualitative and quantitative traits, are chosen as parental genotypes to raise the required mapping population. The strain-specific profiles and pattern similarity within the strains make the method invaluable in addressing problems involved in breeders' rights, genetic homozygosity of the strains, markerassisted breeding and cross-breeding strategies.

\section{Acknowledgements}

JN acknowledges the financial support received from Dept of Biotechnology, New Delhi, and Central Silk Board, Bangalore. 


\section{References}

BECKMANN, J. S. AND WEBER, J. L. 1992. Survey of human and rat microsatellites. Genomics, 12, 627-631.

CHARTERS, Y. M., ROBERTSON, A., WILKINSON, M. J. AND RAMSAY, G. 1996. PCR analysis of oil seed rape cultivars (Brassica oleracea L. ssp. oleifera) using 5'-anchored simple sequence repeat (SSR) primers. Theor. Appl. Genet., 92, 442-447.

EICKBUSH, T. H. 1995. Mobile elements of lepidopteran genomes. In: Goldsmith, M. R. and Wilkins, A. S. (eds) Molecular Model Systems of the Lepidoptera, pp. 77-106. Cambridge University Press, New York.

FIELD, D. AND WILls, C. 1996. Long, polymorphic microsatellites in simple organisms. Proc. R. Soc. B, 263, 209-215.

GOLDSMITH, M. R. 1995. Genetics of the silkworm: revisiting the ancient model system. In: Goldsmith, M. R. and Wilkins, A. S. (eds) Molecular Model Systems of the Lepidoptera, pp. 21-76. Cambridge University Press, New York.

hamada, H., PETRINO, M. AND KAKunaga, T. 1982. A novel repeated element with Z-DNA forming potential is widely found in evolutionarily diverse eukaryotic genomes. Proc. Natl. Acad. Sci. U.S.A., 79, 6465-6469.

KANTETY, R. V., ZENG, X., BENNETZEN, J. L. AND ZEHR, B. 1995. Assessment of genetic diversity in dent and popcorn (Zea mays L.) inbred lines using inter-simple sequence repeat (ISSR) amplification. Mol. Breed., 1, 365-373.

NAGARAJA, G. M. AND NAGARAJU, J. 1995. Genome fingerprinting in silkworm, Bombyx mori, using random arbitrary primers. Electrophoresis, 16, 1633-1638.

NAGARAJU, J., SHARMa, A., SETHURAMAN, B. N., RAO, G. V. AND SINGH, L. 1995. DNA fingerprinting in silkworm Bombyx mori using a banded krait minor satellite DNA-derived probe. Electrophoresis, 16, 1639-1642.

NEI, M. AND LI, W. 1979. Mathematical model for studying genetic variation in terms of restriction endonucleases. Proc. Natl. Acad. Sci. U.S.A., 76, 5269-5273.

PROMBOON, A., SHIMADA, T., FUZIWARA, H. AND KOBAYASHI, M. 1995. Linkage map of random amplified polymorphic DNA (RAPDs) in the silkworm, Bombyx mori. Genet. Res., 66, $1-7$.
Provan, J., POWEll, W. AND WAUgh, R. 1996. Analysis of cultivated potato (Solanum tuberosum) using inter-microsatellite amplification. Genome, 39, 767-769.

ReDDy, K. D., ABRAHAM, E. G. AND NAgARAJu, J. 1999. Microsatellites in the silkworm, Bombyx mori: abundance, polymorphism and strain characterization. Genome, in press.

SCHLOTTERER, C., AMOS, B. AND TAUTZ, D. 1991. Conservation of polymorphic simple sequence loci in cetacean species. Nature, 354, 63-65.

SUZUKI, Y., GAGE, L. AND BROWN, D. D. 1972. The genes for silk fibroin in Bombyx mori. J. Mol. Biol., 70, 637-649.

TAMURA, T., KANDA, T., YAKUHIRO, K., YASKOUCH, N., HINOMOto, N., Shimigu, K. ET AL. 1993. Gamma Field Symposia no. 2, Institute of Radiation Breeding, MAFF, Japan.

TAUTZ, D. AND RENZ, M. 1984. Simple sequences are ubiquitous repetitive components of eukaryotic genomes. Nucl. Acid Res., 12, 4127-4138.

TAUTZ, D., TRICK, M. AND DOVER, G. 1986. Cryptic simplicity in DNA is a major source of genetic variation. Nature, 322, 652-656.

TSUMURA, Y., OHBA, K. AND STRAUSS, S. H. 1996. Diversity and inheritance of inter-simple sequence repeat polymorphisms in Douglas fir (Pseudotsuga menziesii) and sugi (Cryptomeria japonica). Theor. Appl. Genet., 92, 40-45.

WANG, Z., WEBER, J. L., ZHONG, G. AND TANKSLEY, S. D. 1994. Survey of plant short tandem DNA repeats. Theor. Appl. Genet., 88, 1-6.

WEBER, J. L. AND MAY, P. E. 1989. Abundant class of human DNA polymorphisms which can be typed using the polymerase chain reaction. Am. J. Hum. Genet., 44, 388-396.

WU, K. S., JONES, R., DANNEBERGER, L. AND SCOLNIK, P. A. 1994. Detection of microsatellite polymorphisms without cloning. Nucl. Acids Res., 22, 3257-3258.

YAP, I. AND NELSON, R. J. 1996. WinBoot: a program for performing bootstrap analysis of binary data to determine the confidence limits of UPGMA-based dendrograms. IRRI Discussion paper series no. 14, International Rice Research Institute, Manila, Philippines.

ZIETKIEWICZ, E., RAFALSKI, A. AND LABUDA, D. 1994. Genome fingerprinting by simple-sequence repeat (SSR) anchored polymerase chain reaction amplification. Genomics, 20, 176-183. 